\title{
Solving the ternary QCA logic gate problem by means of adiabatic switching
}

\author{
P. Pecar ${ }^{1}$, M. Mraz ${ }^{1}$, N. Zimic ${ }^{1}$, M. Janez ${ }^{1}$ and I. Lebar Bajec ${ }^{1}$ \\ Computer Structures and Systems Laboratory, Faculty of Computer and Information Science, \\ University of Ljubljana, Trzaska 25, 1000 Ljubljana, Slovenia \\ Phone: +386-1-4768-371, Fax: +386-1-4264-647, E-mail: primoz.pecar@ fri.uni-lj.si
}

\section{INTRODUCTION}

Quantum-dot cellular automata (QCA) [1] are one of the most promising alternative platforms of the future. Recent years have witnessed the development of basic logic structures as well as more complex processing structures, however all in the realm of binary logic. On the other hand Lebar Bajec et al. [2] have, on the grounds that future platforms should not disregard the advantages of multi-valued logic [3], showed that QCA can be used for the implementation of ternary logic as well. In their solution the building block that proved as the most troublesome was the one that implements the ternary AND and OR logic functions. In this study we present a solution of the problem that is based on adiabatic switching.

\section{EXPERIMENTS}

According to Lebar Bajec et al., a ternary QCA cell is a planar structure comprising eight quantum dots and two electrons that can tunnel between neighbouring dots (see Fig. 1). With this cell, which has four distinctive but equivalent ground states marked as A, B, C and D (see Fig. 2), they represent three logic values. They show that the structures that implement the QCA line and inverter retain their functionality with a simple switch of the basic building block (i.e. binary cell for its ternary counterpart). This, however, was not true for the geometry of the structure that implements the AND and OR logic gates (see Fig. 3). The authors solved the problem with an augmented structure [2] that is, from the space requirement perspective, not well-chosen, as its size is three times larger than the comparable binary one, even if one disregards the interconnections.

We here report a solution that is based on adiabatic switching [3]. We first needed to develop a new simulation model, as the semi-classical approach [5], used by Lebar Bajec et al., does not support modelling adiabatic switching. The ternary QCA cell was thus described with a quantummechanical model that is based on the Hamiltonian equation of the Hubbard type. The values of the employed constant parameters were taken as if the material in use was GaAs.

The adiabatic switching ensures the modelled structure is in its ground state throughout the whole time of the switch. This is achieved by controlling the interdot barriers. They are controlled by means of a cyclic signal consisting of four phases of equal length (see Fig 4). By taking this into account, an arbitrary QCA structure can be decomposed into multiple parts controlled by distinct 90deg phase shifted signals (see Fig. 5). Using this approach we decomposed the challenging geometry from figure 3 into three subsections. The first one comprises the input cells denoted $S, X_{1}$ and $\mathrm{X}_{2}$, the second the internal cell and the third the output cell $\mathrm{Y}$, as presented in figure 5. It turns out that in such a case the geometry works as intended, i.e. as ternary logic AND and OR gates by means of a simple switch of the basic building block (i.e. binary cell for its ternary counterpart).

\section{SUMMARY}

We have shown that with the introduction of adiabatic switching one can successfully solve the ternary QCA problem of the basic geometry that implements the AND and OR logic functions. What is more, we have shown that in that case the geometry can perform both the binary majority logic function as well as ternary AND and OR logic functions.

\section{REFERENCES}

[1] C.S. Lent, P.D. Tougaw, W. Porod, G.H. Bernstein, Nanotechnology 4, 49 (1993).

[2] I. Lebar Bajec, N. Zimic, M. Mraz, Nanotechnology 17, 1937 (2006).

[3] B. Hayes, Am. Sci. 89, 490 (2001).

[4] P.D. Tougaw, C.S. Lent, J. Appl. Phys. 80, 4722 (1996).

[5] M. Macucci, G. Iannaccone, S. Francaviglia, B. Pellegrini, Int. j. circuit theory appl. 29, 37 (2001). 


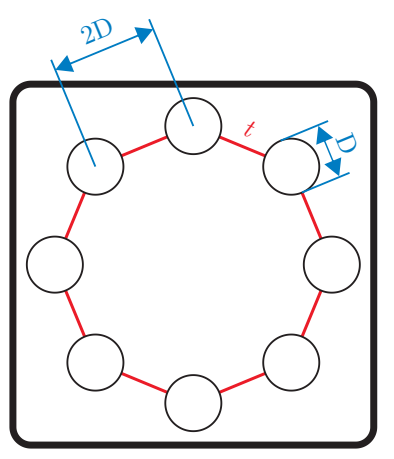

Fig. 1 Geometry of ternary quantum-dot cell. It is comprised of eight quantum dots with diameter $D$, which are occupied by two electrons. The nearneighbour distance between dot centers is $2 D$. Red lines denote tunneling paths, where $t$ is the tunneling energy.

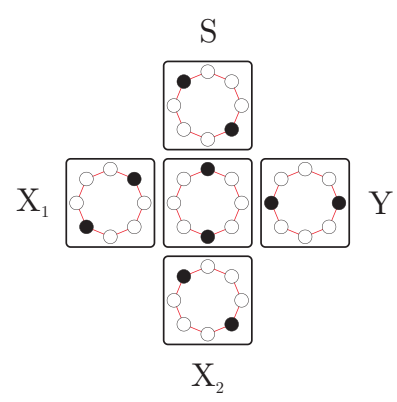

\begin{tabular}{|c|c|c|c|c|}
\hline & $\mathrm{S} \mathrm{X}_{1}$ & & $\mathrm{~S} \mathrm{X}_{1} \mathrm{X}_{2} \mathrm{Y}$ & $\mathrm{Y}$ \\
\hline & $\begin{array}{ll}0 & 0\end{array}$ & & $\mathrm{AAAA}$ & 0 \\
\hline & $\begin{array}{ll}0 & 0\end{array}$ & $1 / 2$ & A A C A & 0 \\
\hline & $\begin{array}{ll}0 & 0\end{array}$ & 1 & A A B A & 0 \\
\hline & $0^{1 / 2}$ & 0 & A C A A & 0 \\
\hline & $0^{1 / 2}$ & $1 / 2$ & A C C C & $1 / 2$ \\
\hline & $0^{1 / 2}$ & 1 & $\mathrm{AC} \mathrm{B} \mathrm{C}$ & $1 / 2$ \\
\hline & $\begin{array}{ll}0 & 1\end{array}$ & 0 & A B A D & 0 \\
\hline & $\begin{array}{ll}0 & 1\end{array}$ & $1 / 2$ & A B C C & $1 / 2$ \\
\hline & $\begin{array}{lll}0 & 1\end{array}$ & 1 & A B B B & 1 \\
\hline & 10 & 0 & B A A A & 0 \\
\hline & 10 & $1 / 2$ & B A C C & $1 / 2$ \\
\hline & 10 & 1 & B A B D & 1 \\
\hline & $1 \frac{1 / 2}{2}$ & 0 & B C A C & $1 / 2$ \\
\hline & $1 \frac{1 / 2}{2}$ & $1 / 2$ & B C C C & $1 / 2$ \\
\hline & $1 \frac{1 / 2}{2}$ & 1 & B C B B & 1 \\
\hline & 11 & 0 & B B A B & 1 \\
\hline & 11 & $1 / 2$ & B B C B & 1 \\
\hline & 11 & 1 & B B B B & 1 \\
\hline
\end{tabular}

Fig. 3 The problematic structure comprised of three input cells $\mathrm{S}, \mathrm{X}_{1}$ and $\mathrm{X}_{2}$, one internal cell and one output cell $\mathrm{Y}$. As we can see in the table the structure behaves almost as the ternary AND or OR logic gates. There are two erroneous outputs (ie. $\operatorname{AND}(1,0)$ and $\mathrm{OR}(0,1))$.

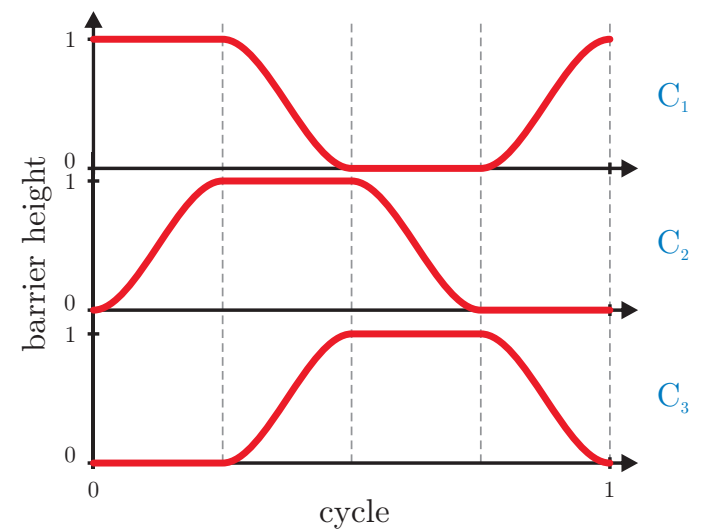

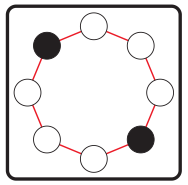

A

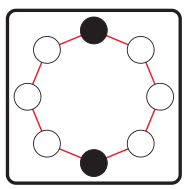

C

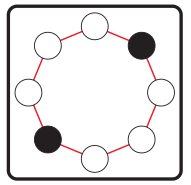

B

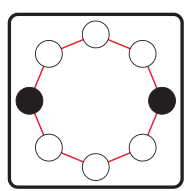

D
Fig. 2 The four distinctive but equivalent ground states of the ternary QCA cell. State A is interpreted as logical value 0 , state B as logical value 1 , state $C$ as logical value $1 / 2$. State $D$ is allowed only as an internal processing state and it can not be interpreted as a logical value.

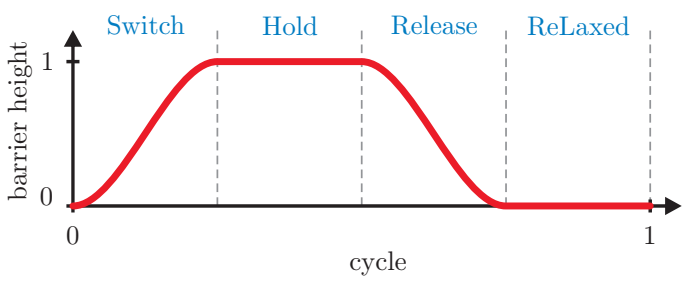

Fig. 4 The cyclic signal that controls interdot barriers has four phases. The barrier heights are normalized. The value 0 corresponds to low barriers; the confinemet of the electrons on the individual quantum dots is reduced. The value 1 corresponds to high barriers, which localizes the electrons on the individual quantum dots.

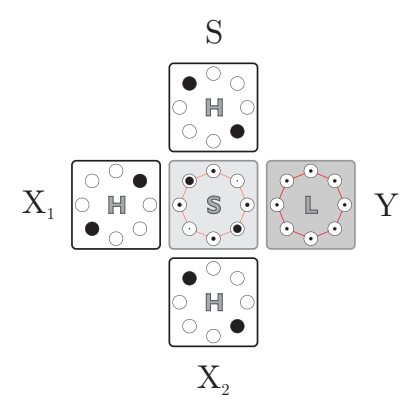

Fig. 5 The geometry is decomposed into three subsections, controlled by three phase shifted cyclic signals, denoted $\mathrm{C}_{1}, \mathrm{C}_{2}, \mathrm{C}_{3}$. Signal $\mathrm{C}_{1}$ controls the input cells $\mathrm{S}, \mathrm{X}_{1}, \mathrm{X}_{2}$, signal $\mathrm{C}_{2}$ controls the internal cell and signal $\mathrm{C}_{3}$ controls the output cell. In this case the geometry works as intended, i.e. as ternary logic AND if either $\mathrm{S}, \mathrm{X}_{1}$ or $\mathrm{X}_{2}$ is in state $\mathrm{A}$ and as ternary logic OR if either $\mathrm{S}, \mathrm{X}_{1}$ or $\mathrm{X}_{2}$ is in state $\mathrm{B}$. 